\title{
Sequential drug delivery by injectable macroporous hydrogels for combined photodynamic-chemotherapy
}

\author{
Yuanyuan Zhong ${ }^{1 \dagger}$, Li Zhang ${ }^{1 \dagger}$, Shian Sun ${ }^{3}$, Zhenghao Zhou' , Yunsu Ma ${ }^{1}$, Hao Hong ${ }^{2^{*}}$ and Dongzhi Yang ${ }^{1^{*}}$ (1)
}

\begin{abstract}
With hollow mesoporous silica (hMSN) and injectable macroporous hydrogel (Gel) used as the internal and external drug-loading material respectively, a sequential drug delivery system DOX-CA4P@Gel was constructed, in which combretastatin A4 phosphate (CA4P) and doxorubicin (DOX) were both loaded. The anti-angiogenic drug, CA4P was initially released due to the degradation of Gel, followed by the anti-cell proliferative drug, DOX, released from hMSN in tumor microenvironment. Results showed that CA4P was mainly released at the early stage. At $48 \mathrm{~h}, \mathrm{CA} 4 \mathrm{P}$ release reached $71.08 \%$, while DOX was only $24.39 \%$. At 144 h, CA4P was $78.20 \%$, while DOX release significantly increased to $61.60 \%$, showing an obvious sequential release behavior. Photodynamic properties of porphyrin endow hydrogel $\left(\varphi_{\Delta(G e l)}=0.91\right)$ with enhanced tumor therapy effect. In vitro and in vivo experiments showed that dual drugs treated groups have better tumor inhibition than solo drug under near infrared laser irradiation, indicating the effectivity of combined photodynamic-chemotherapy.
\end{abstract}

Keywords: Sequential drug delivery, Combretastatin A4 phosphate, Doxorubicin, Photodynamic therapy, Combined photodynamic-chemotherapy

\section{Introduction}

Cancer is still one of the significant threats to human health. Current cancer therapies, such as chemotherapy [1], phototherapy [2], hyperthermia [3] and radiotherapy [4], are widely applied but they have their own limited properties or unavoidable side effects. For example, patients always suffer from serious side effects by intrinsic off-target toxicity of chemotherapy drugs, also endure the possible low effective treatment for the limited relatively short resident time in tumor cells [5]. Phototherapy and hyperthermia against deep tumors may greatly limited by insufficient luminous

\footnotetext{
*Correspondence: haohong@nju.edu.cn; dongzhiy@xzhmu.edu.cn †Yuanyuan Zhong and Li Zhang contributed equally to this work 1 Jiangsu Key Laboratory of New Drug Research and Clinical Pharmacy, Xuzhou Medical University, Xuzhou 221004, Jiangsu, China

2 Medical School, Nanjing University, Nanjing 210093, Jiangsu, China

Full list of author information is available at the end of the article
}

flux [6]. Radiotherapy can also damage nearby healthy cells beside killing cancer cells [7]. Especially for chemotherapy, the drug's resident time in tumor cells is relatively short, which limited the theragnostic effect. Therefore, it's necessary to develop local sequential delivery of multiple drugs for optimal efficacy, potency, and safety. The co-administration of dual drugs with different physicochemical properties and specific administration sequence is very important to overcome drug resistance and reduce side effects in tumor therapy. Anti-cell proliferative drugs, such as doxorubicin (DOX) and paclitaxel (PTX), can kill cancer cells from different proliferation stages. However, due to the support of peripheral blood vessels, the survived tumor cells could still obtain nutrition supply and continue to proliferate [8]. Anti-angiogenic drugs can specifically target vascular growth factors to inhibit the establishment of peri-tumoral blood vessels [9]. CA4P is one of 
novel angiogenesis inhibitors, which can induce apoptosis by binding tubulin dimers and preventing microtubule polymerization. It's reported that CA4P has the potential to sensitize drug-resistant MCF-7/ADR cells to DOX due to the inhibition of angiogenesis [10]. Anti-angiogenesis combined with anti-cell proliferation has become a novel tumor therapy strategy, which was referred as "A+ strategy" [11]. When both drugs were applied to the tumor site at the right time, they could maximize the therapeutic effect. We hoped to design a system that anti-angiogenic drugs could be released firstly to inhibit the growth of new tumor vasculatures, while anti-cell proliferative drugs could be slowly released and accumulated in the tumor site to effectively kill tumor cells.

Hydrogel is composed of a large amount of water and cross-linked polymers. The abundant water content (7099\%) in hydrogels resembles biological tissues, and they usually have good biocompatibility and loading hydrophilic drugs capacity [12]. Smart hydrogels (temperature responsive [13], pH responsive [14], light responsive [15], or NO responsive [16]) were frequently used in controlled drug release studies due to their unique properties. For example, Kim et al. [17] prepared a hydrogel/ microsphere composite, which could sequentially release doxorubicin (DOX) and fluorouracil (Fu) in tumors. Fu was directly encapsulated in the hydrogel, and DOX was loaded in microspheres. Results showed that $80 \% \mathrm{Fu}$ was released at $72 \mathrm{~h}$, while the release of DOX only reached about $40 \%$, proving that the smart composite hydrogel with nano-sized microspheres could be used as a carrier for the sequential release of different drugs.

To further enhance the therapeutic effect of drugs, photodynamic therapy (PDT) has been used in combination with chemotherapy, magnetic therapy or immunity therapy because of its significant advantages such as causing less trauma, low toxicity, and palliative treatment [18]. Belali et al. [19] used amino-modified 5,10,15,20-tetraphenylporphyrin (TPP) to prepare $\mathrm{pH}$-sensitive hydrogels which could achieve efficient photodynamic therapy effects. Gonzalez-Delgado et al. [20] found that hydrogels enhanced singlet oxygen generation efficiency of porphyrin derivatives when they were encapsulated in hydrogels.

Herein, we designed and prepared a novel injectable macroporous hydrogel for therapy of $4 \mathrm{~T} 1$ breast cancer, which could load dual distinct tumor drugs (CA4P and DOX) offering a sequentially delivery profile. Furthermore, porphyrin derivatives in hydrogel trigger the formation of reactive oxygen species (ROS), achieving synergistic anticancer effects in photodynamic-chemotherapy combination therapy. The therapeutic efficacy from this combinational photodynamic/sequential chemotherapy was evaluated in vitro and in vivo.

\section{Experimental section}

\section{Chemical and reagents}

Tetraethyl silicate (TEOS), triethanolamine (TEA), 5,10,15,20-tetraphenylporphyrin (TPP), (5,10,15,20)-tetra(4aminophenyl)porphyrin $\left(\mathrm{NH}_{2}-\mathrm{TPP}\right)$, dextran and doxorubicin hydrochloride (DOX $\cdot \mathrm{HCl})$ were all purchased from Shanghai Aladdin Biochemical Technology Co., Ltd. 3-Aminopropyltrimethoxysilane (APS) was purchased from Braunwell Chemical Technology Co., Ltd. CA4P was acquired from Solarbio Science \& Technology Co., Ltd. Cetyltrimethylammonium chloride (CTAC) was purchased from Sigma-Aldrich Co., Ltd. Dulbecco's modified Eagle's medium (DMEM), memorial institute medium (RPMI)1640, 3-(4,5-dimethylthiazol-2-yl)-2,5-diphenyltetrazolium bromide (MTT), 4',6-diamidino-2-phenylindole (DAPI), 1,3-diphenylisobenzofuran (DPBF), 2',7'-dichlorodihydrofluorescein diacetate (DCFH-DA), live/dead cell viability/ toxicity kit, and Annexin V-FITC/PI staining kit were all purchased from Vicmed (Xuzhou, China). Caspase 3 polyclonal antibody (caspase $3 \mathrm{PAb}$ ) was purchased from Proteintech Co. (USA). PV-6001 rabbit polymer detection system was purchased from Zhongshan Jinqiao Co., Ltd. Trypsin and dimethyl sulfoxide (DMSO) were purchased from Nanjing Key Gen Biotechnology Inc. Dextran (W=70 $\mathrm{kD})$, chitosan with medium viscosity $(200-400 \mathrm{mPa} \cdot \mathrm{s})$, and sodium periodate were purchased from Macklin Biochemical Technology Co., Ltd. Other reagents were all purchased from Sinopharm Group Chemical Reagent Co., Ltd.

\section{Synthesis of DOX-CA4P@Gel}

Hollow mesoporous silica nanoparticle (hMSN) was prepared according to our previous work [21]. Briefly, anhydrous ethanol, double distilled water and $25 \%$ ammonia $(\mathrm{V}: \mathrm{V}: \mathrm{V}=180: 25: 4)$ were mixed and stirred for $10 \mathrm{~min}$, followed by adding TEOS to acquire silica nanoparticles $\left(\mathrm{dSiO}_{2}\right)$. After washing with water, $\mathrm{dSiO}_{2}$ was added into the mixed solution of CTAC and TEA, followed by drop-wise adding TEOS and the reaction was carried out at $80{ }^{\circ} \mathrm{C}$ for $1 \mathrm{~h}$ to synthesize $\mathrm{dSiO}_{2} @ \mathrm{SiO}_{2} \cdot \mathrm{Na}_{2} \mathrm{CO}_{3}$ powder was then added to it to allow etching $\mathrm{dSiO}_{2} @ \mathrm{SiO}_{2}$ at $50{ }^{\circ} \mathrm{C}$ for $30 \mathrm{~min}$. After washing with $\mathrm{NaCl}$ :methanol (1\%), hMSN was achieved. Finally, hMSN was modified with amino groups on the surface via hydrolyzing APS in absolute ethanol.

Hydrogel OD/TPP was synthesized through a Schiff base reaction between the aldehyde groups from oxidized dextran (OD) and amino groups of porphyrins with the molar ratio of 1:10. Firstly, the adjacent diol groups were oxidized to aldehyde groups by sodium periodate to prepare aldehydated oxidized dextran [22]. The oxidation degree of aldehyde groups (proportion of oxidized dextran repeating units) was determined by measuring the aldehyde content via a hydroxylamine hydrochloride 
titration method with bromophenol blue as the indicator [23]. Secondly, $10 \mathrm{mg}$ oxidized dextran (dissolved in $1 \mathrm{~mL}$ PBS with $\mathrm{pH}$ value of 6.8) was mixed with $1 \mathrm{mg}$ $\mathrm{NH}_{2}$-TPP (dissolved in $1 \mathrm{~mL}$ DMF) to form OD/TPP, the unreacted OD and TPP was removed using dialysis bags (MWCO 12000-14000) against distilled water for 3 days (the water was renewed at least 5 times per day). $30 \mathrm{mg}$ chitosan (dissolved in 1\% acetic acid) was applied to adjust the gelation level of OD/TPP (with the mass ratio of TPP:OD:chitosan of 10:1:30) to form 'hydrogel'. hMSN was loaded in hydrogel by simply mixing during the gelation of OD/TPP, the hydrogel loaded hMSN was referred as 'Gel'. The excessive and unreacted reagents were removed by washing with distilled water based on the 'no flow' status of hydrogel.

DOX and CA4P was loaded into Gel in different methods to synthesize the composite system DOX-CA4P@ Gel. Briefly, DOX $\cdot \mathrm{HCl}$ aqueous solution was adjusted to $\mathrm{pH}$ 8.0, followed by mixing with hMSN at a mass ratio of 1:1. The mixture was stirred for $24 \mathrm{~h}$ at room temperature and the excess DOX was removed by dialyzing with molecular weight cut-off of $2 \mathrm{kDa}$ to acquire drug-loaded microspheres DOX@hMSN [24]. The mixture of DOX@ hMSN and $\mathrm{NH}_{2}$-TPP with a mass ratio of $1: 5$ was dissolved in DMF to acquire system 'A', and CA4P was mixed with oxidized dextran at a ratio of 1:1 (in PBS) to prepare system 'B'. DOX-CA4P@Gel was synthesized through a Schiff base reaction between $A$ and $B$. The unreacted reagents were removed by washing with distilled water.

\section{Characterization}

The gelation time and the degradation behavior of the hydrogel under different acidic conditions were detected by an MCR 302 rheometer. Transmission electron microscopy (TEM) images were taken on a G2T12 transmission electron microscope (FEI, USA). The morphology of the hydrogel was recorded on a Q25 scanning electron microscope (FEI, USA). The hydrodynamic size and zeta potentials were measured by dynamic light scattering (DLS) (380 ZLS, NICOMP, USA). The chemical compositions were determined by X-ray photoelectron spectroscopy (XPS) (PHI Quantera SXM). All XPS spectra were calibrated by the $\mathrm{C} 1 \mathrm{~s}$ peak at $284.6 \mathrm{eV}$. Infrared absorption spectrum (IR) was recorded on a FTIR 8400 spectrophotometer. UV-Vis and fluorescence spectra were recorded on Hitachi U-3010 and F-4600 spectrophotometer, respectively.

\section{Drug release measurement}

Drug release behavior was evaluated at $37^{\circ} \mathrm{C}$ by respectively incubating DOX-CA4P@Gel in PBS solution with $\mathrm{pH}$ of 5.0, 6.4, and 7.2. CA4P release was measured by high performance liquid chromatograph (HPLC), where chromatographic separation was carried out on an Agilent Zorbax Eclipse Plus C18 column with particles size of $5.0 \mu \mathrm{m}(250 \times 4.6 \mathrm{~mm})$ using methanol $(0.01 \%$ acetic acid)-water (60:40) as the mobile phase at $1 \mathrm{~mL} / \mathrm{min}$ [25]. The detection wavelength was $305 \mathrm{~nm}$. DOX release was calculated by determining unbound DOX in the solution with UV-vis spectrometry [26].

\section{Evaluation of singlet oxygen generation efficiency}

The optical property of DPBF was sensitively affected by ${ }^{1} \mathrm{O}_{2}$ [27]. With DPBF as the ${ }^{1} \mathrm{O}_{2}$ capture agent, the ${ }^{1} \mathrm{O}_{2}$ generation efficiency of TPP in Gel can be calculated from the slopes of DPBF absorbance variation $(410 \mathrm{~nm})$ in the presence of photosensizers. The laser irradiation was $808 \mathrm{~nm}\left(0.5 \mathrm{~W} / \mathrm{cm}^{2}\right)$, with TPP in DMSO as the control, the ${ }^{1} \mathrm{O}_{2}$ yield $\left(\phi_{\Delta}\right)$ was calculated as follows [3]:

$\phi_{\Delta(\mathrm{Gel})}=\phi_{\Delta(\mathrm{TPP})} \frac{t_{\mathrm{TPP}}}{t_{\mathrm{Gel}}}$ Where $\phi_{\Delta(\mathrm{Gel})}$ represents the singlet oxygen yield of Gel, $\phi_{\Delta(\mathrm{TPP}) \text { was }} 0.52 . t_{\mathrm{TPP}}$ and $t_{\mathrm{Gel}}$ is the time for the decrease in absorption of DPBF in the presence of TPP and Gel, respectively.

\section{Cell culture and animal model}

4T1 breast cancer cells and L929 fibroblasts cells were maintained in RPMI 1640 and DMEM medium supplemented with $10 \%$ fetal bovine serum and proper antibiotics at $37{ }^{\circ} \mathrm{C}$ with the supply of $5 \% \mathrm{CO}_{2}$. Cells were used when they reached $\sim 80 \%$ confluence.

All animal procedures were performed in accordance with the National Academy of Sciences' Guidelines on the Care and Use of Laboratory Animals [28], and were approved by the Animal Ethics Committee of Xuzhou Medical University (L20210226103). Tumors were established by subcutaneous injection of $1 \times 10^{6}$ of $4 \mathrm{~T} 1$ cells suspended in $50 \mu \mathrm{L}$ of PBS into female Balb/c mice (5-week-old, 18-20 g). Mice were used for therapy study when the tumor diameter reached $5-8 \mathrm{~mm}$, monitored every other day by a caliper measurement.

\section{Fluorescence microscopy and flow cytometry}

MTT assays were carried to evaluate the biocompatibility (without laser irradiation) and the therapeutic efficacy (with laser irradiation) of DOX-CA4P@Gel. DOX-CA4P@Gel at the concentration of $0.01-0.20 \mathrm{mg} /$ $\mathrm{mL}$ were respectively incubated with $4 \mathrm{~T} 1$ and $\mathrm{L} 929$ cells for 24,48 , and $72 \mathrm{~h}$. MTT measurement was subsequently carried out to evaluate the cellular toxicity of DOX-CA4P@Gel [29]. To assist the study of the release behavior for CA4P and DOX, MTT assay was used to detect $4 \mathrm{~T} 1$ cells viability under different incubation conditions, including different media with $\mathrm{pH}$ values of 5.0, 6.4 and 7.2, with/without laser irradiation $(808 \mathrm{~nm}$, $0.5 \mathrm{~W} / \mathrm{cm}^{2}$, and $5 \mathrm{~min}$ ). All experiments were performed at least three times. Five groups including CA4P@Gel, 
DOX@Gel, DOX-CA4P@Gel, DOX and CA4P were compared in this work.

The fluorescence microscopy was used to observe the production efficiency of ${ }^{1} \mathrm{O}_{2}$ and cytotoxic effect of DOX-CA4P@Gel in 4T1 cells.4T1 cells were cultured at a 24-well plate with concentration of $5 \times 10^{4}$ cells/ $\mathrm{mL}$. After incubation with hydrogel extracted solution $(0.5 \mathrm{mg} / \mathrm{mL}$ based on TPP) for $24 \mathrm{~h}$ with laser irradiation $\left(808 \mathrm{~nm}, 0.5 \mathrm{~W} / \mathrm{cm}^{2}\right.$, and $5 \mathrm{~min}$ ), the cells were stained with DCFH-DA and DAPI [30]. Cells were analyzed using fluorescence microscopy. Furthermore, the cells were also stained with live/dead staining kits to visually examine the killing effect of DOX-CA4P@Gel under laser irradiation [31].

MACSQuant 10 flow cytometry (Miltenyi Biotec, GER) was also used to study the drug release behavior of CA4P and DOX from DOX-CA4P@Gel. 4T1 cells were dispersed with a final concentration of $1 \times 10^{5} /$ well. CA4P@ Gel, DOX@Gel, DOX-CA4P@Gel extracts at different concentrations were incubated with $4 \mathrm{~T} 1$ cells for $24 \mathrm{~h}$ with or without laser irradiation $\left(808 \mathrm{~nm}, 0.5 \mathrm{~W} / \mathrm{cm}^{2}\right.$, and $5 \mathrm{~min}$ ). Flow cytometry assay was carried out after Annexin V-FITC $5 \mu \mathrm{L}$ and PI $5 \mu \mathrm{L}$ staining for $10 \mathrm{~min}$.

\section{In vivo therapeutic evaluation}

The mice bearing $4 \mathrm{~T} 1$ tumor nodules were randomly divided into 10 groups $(\mathrm{n}=8)$ including PBS (Control), free DOX/CA4P (mixture of $0.4 \mathrm{mg}$ DOX and $1 \mathrm{mg}$ CA4P), Gel, CA4P@Gel, DOX@Gel, DOX-CA4P@ Gel, Gel+NIR (near infrared laser), CA4P@Gel+NIR, DOX@Gel+NIR, and DOX-CA4P@Gel+NIR. $100 \mu \mathrm{L}$ of each sample were intravenously injected into tumor. Except for PBS and free DOX/CA4P, the concentrations of hydrogel were consistent $(44 \mathrm{mg} / \mathrm{mL})$ in eight groups. During preparation of CA4P@Gel and DOX@ Gel, proper amount of DOX and CA4P were added to ensure the consistent concentration in DOX-CA4P@ Gel. An $808 \mathrm{~nm}$ laser with $0.5 \mathrm{~W} / \mathrm{cm}^{2}$ power intensity was used as the near-irradiation source. During therapy, the body weight and tumor volume $\left(V=L \times W^{2} / 2\right)$ were monitored every other day, where $L(\mathrm{~mm})$ and $W(\mathrm{~mm})$ are the long and short axes of tumor size, respectively. The tumor inhibition rate (TIR) was calculated using the equation of TIR $=\left(V_{\mathrm{c}}-V_{\mathrm{t}}\right) / V_{\mathrm{c}} \times 100 \%$, where $V_{\mathrm{t}}$ and $V_{\mathrm{c}}$ represent the tumor volumes in the treatment group and control group, respectively. After treatment, major organs including heart, liver, spleen, lung, and kidney were harvested for the hematoxylin and eosin (H\&E) analysis. The 4T1 tumors harvested on day $0,3,7$, and 14 were also frozen for histological analysis.

\section{Histology}

Major organs were fixed in $4 \%$ formalin, transferred routinely into paraffin, sectioned into $6 \mu \mathrm{m}$ thick slices, stained with H\&E and examined by a microscopy with a magnitude of $200 \times$. Tumors were cut into frozen slices of $6 \mu \mathrm{m}$ thickness. After being fixed with cold acetone for $10 \mathrm{~min}$, the tumor slides were stained with caspase 3 PAb (catalog number: 19677-1-AP) for $1 \mathrm{~h}$, followed by detecting by PV-6001 rabbit kit. The caspase 3 expression was by a confocal microscope at a magnitude of $200 \times$.

\section{Results and discussion}

\section{Preparation and characterization of Gel conjugation}

As shown in Fig. 1a, hydrogel was obtained by a one-step reaction among $\mathrm{OD}$, chitosan and $\mathrm{NH}_{2}$-TPP, in which the OD-to- $\mathrm{NH}_{2}$-TPP ratio was $1: 10$, and chitosan (W\% = $10 \%)$ was to adjust the elasticity. The degree of aldehyde was closely related to the oxidation time by sodium periodate. Considering the reaction time and the degree of aldehyde (Additional file 1: Table S1), the optimum oxidation time was $16 \mathrm{~h}$. The amount of chitosan affected the formation and degradation of hydrogel, results showed in Additional file 1: Table S2 indicated that 73\% ( $w_{\text {chi- }}$ osan $\%)$ was optimal for getting fast formation and slowly degradation. As shown in Fig. 1b, the disappearance of characteristic IR absorbance peaks from oxidized dextran (at $\sim 1712 \mathrm{~cm}^{-1}$ for $\mathrm{C}=\mathrm{O}$ ) and porphyrin derivative $\left(\sim 1510 \mathrm{~cm}^{-1}\right.$ for $\left.\mathrm{N}-\mathrm{H}\right)$, and presence of characteristic IR peak from Gel $\left(\sim 1650 \mathrm{~cm}^{-1}\right.$ for $\left.\mathrm{C}=\mathrm{N}\right)[3,19]$ confirm the successful reaction between OD and TPP. The gel-forming behavior under physiological conditions was also evaluated by measuring the storage modulus $\left(\mathrm{G}^{\prime}\right)$ and loss modulus $\left(G^{\prime \prime}\right)$, where the sol-gel conversion condition could be determined. As shown in Fig. $1 \mathrm{c}$ and d, the conversion occurred at $\sim 36.5^{\circ} \mathrm{C}$ for $\mathrm{Gel}$, and $\sim 36.8^{\circ} \mathrm{C}$ for DOX-CA4P@Gel, which indicated that loading of CA4P and DOX did not change the gelation process of TPP, chitosan, and OD. Gel could form within $8.8 \mathrm{~min}$, and DOXCA4P@Gel formed within $8.5 \mathrm{~min}$. Results showed that the hydrogel can be synthesized quickly at a temperature close to physiological temperature, indicating its injectability in vivo.

As shown in Fig. 2a, DOX was loaded into hMSN to form DOX@hMSN, and then DOX@hMSN was enveloped by OD/TPP together with CA4P to form dual drugs loaded DOX-CA4P@Gel. The morphology and size distribution histogram of hMSN was shown in Fig. 2b. The average diameter of hMSN was $120 \mathrm{~nm}$ from TEM assay, smaller than that measured from DLS (with the average diameter of $142 \mathrm{~nm}$ ) for hydration effects. The zeta potential analysis was constructed in Additional file 1: Figure S1. Compared to 


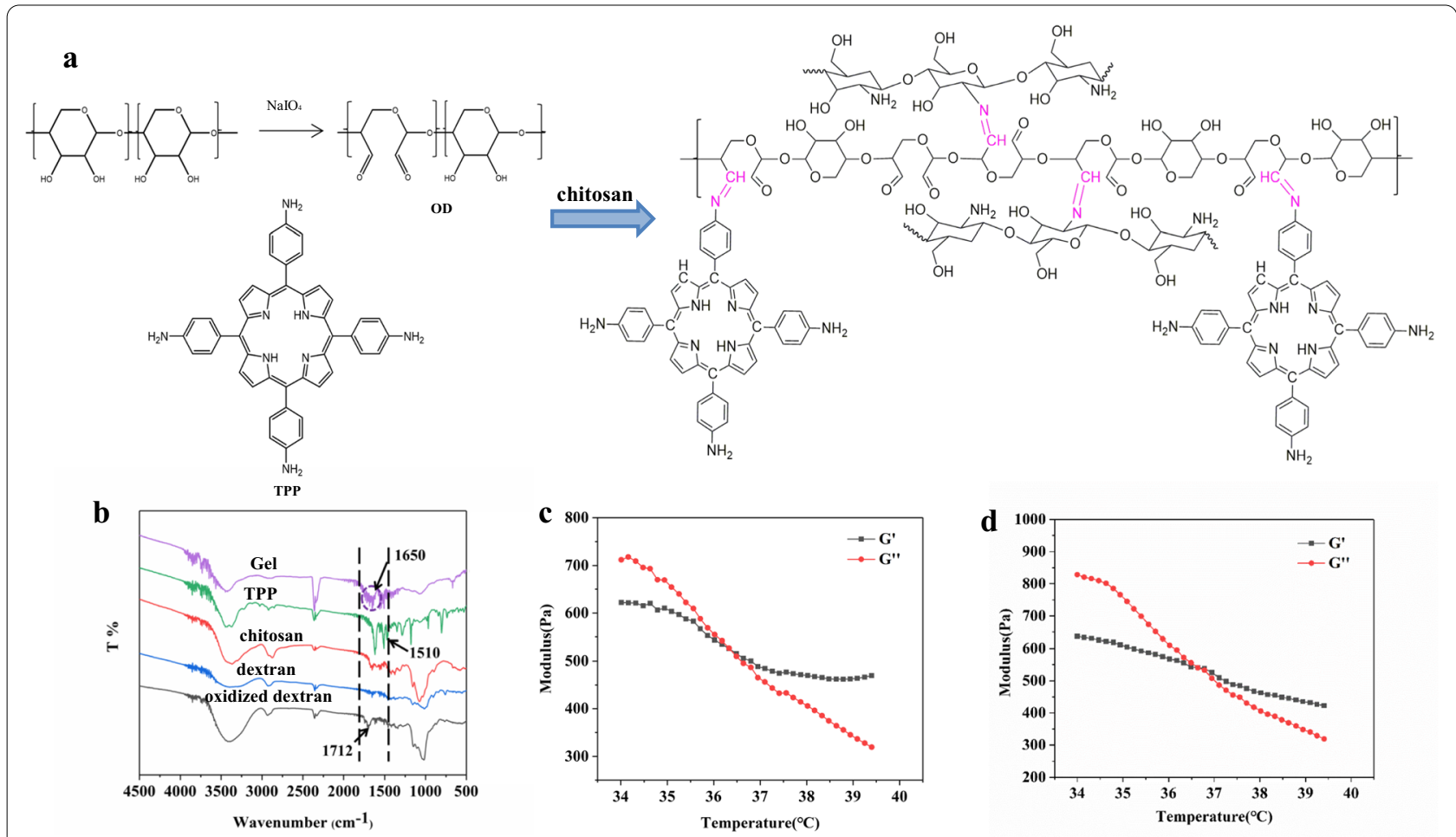

Fig. 1 Schematic structural information (a), IR spectra (b), the storage modulus and loss modulus of OD/TPP hydrogel (c) and DOX-CA4P@Gel (d)

the value of hMSN $(-29.8 \pm 1.1 \mathrm{mV})$, the zeta potentials of hMSN-NH $\mathrm{N}_{2}$ significantly increased, indicating a modification effect of amino groups. No obvious difference was present between DOX-CA4P@Gel (38.4 $\pm 3.8 \mathrm{mV})$ and CA4P@Gel $(33.5 \pm 2.4 \mathrm{mV})$. Figure 2c, $\mathrm{d}$ was SEM images of the lyophilized cross-section of the hydrogel. Results showed that the hydrogel possessed a three-dimensional network structure with a uniform pore size of about $5 \mu \mathrm{m}$. Figure $2 \mathrm{e}-\mathrm{g}$ showed the three-dimensional network structure of the freezedried cross-section of DOX-CA4P@Gel. The results showed that DOX@hMSN did not affect the morphology of the hydrogel network structure, and the bulges in the three-dimensional structure indicated the locations of hMSN. The surface chemical compositions of the hydrogel layers from XPS also confirmed it. Comparing the X-ray photoelectron spectrum of Gel with that of DOX-CA4P@Gel (Additional file 1: Figure S2), the peaks seen at $154.1 \mathrm{eV}$ and $102.5 \mathrm{eV}$ indicate that there were additional Si elements on the surface of Gel, which were resourced from hMSN. According to the absorbance of DOX at $510 \mathrm{~nm}$, where TPP and CA4P didn't affected its detection (Additional file 1: Figure S3), the drug loading capacity of DOX in hMSN was $633 \mathrm{mg} / \mathrm{g}$, and $52 \mathrm{mg} / \mathrm{g}$ for DOX in DOX-CA4P@Gel.
The drug loading of CA4P in hydrogel was $12.6 \mathrm{mg} / \mathrm{g}$ calculating from HPLC measuring.

\section{Drug sequential release from DOX-CA4P@Gel}

DOX-CA4P@Gel was dispersed in phosphate buffer solution (PBS) solution with different $\mathrm{pH}$ values, and the elastic modulus of hydrogel was measured using a rheometer. As shown in Fig. 3a, b and c, the energy storage modulus $\left(\mathrm{G}^{\prime}=34,000\right)$ of hydrogels at $\mathrm{pH}$ of 5.0 was significantly higher than that in $\mathrm{pH} 7.2\left(\mathrm{G}^{\prime}=17,000\right)$, which proved that the hardness of hydrogels under acidic conditions was more than that in neutral condition, and the hydrogels were more prone to fracture under the same shear force. This result was consistent with the corresponding crushing time $\left(t_{\mathrm{pH} 5.0}=5.0 \mathrm{~min}, t_{\mathrm{pH} 6.4}=5.2 \mathrm{~min}, t_{\mathrm{pH}}\right.$ $7.2=6.4 \mathrm{~min})$. DOX-CA4P@Gel was dispersed in PBS with different $\mathrm{pH}$ values, and the degradation of hydrogels was evaluated by measuring the content of decomposed TPP in solution. Results in Fig. 3d showed that the degradation rate of hydrogels was very slow in neutral condition (about $20 \%$ at $144 \mathrm{~h}$ ), while that was significantly increased in weakly acid condition, reaching $65 \%$ at $144 \mathrm{~h}$ in $\mathrm{pH}$ of 6.4. Degradation of hydrogel at $\mathrm{pH}$ 5.0 was faster and more than $90 \%$ TPP was detected at $144 \mathrm{~h}$. This result testified that $\mathrm{pH}$ sensitive degradation 

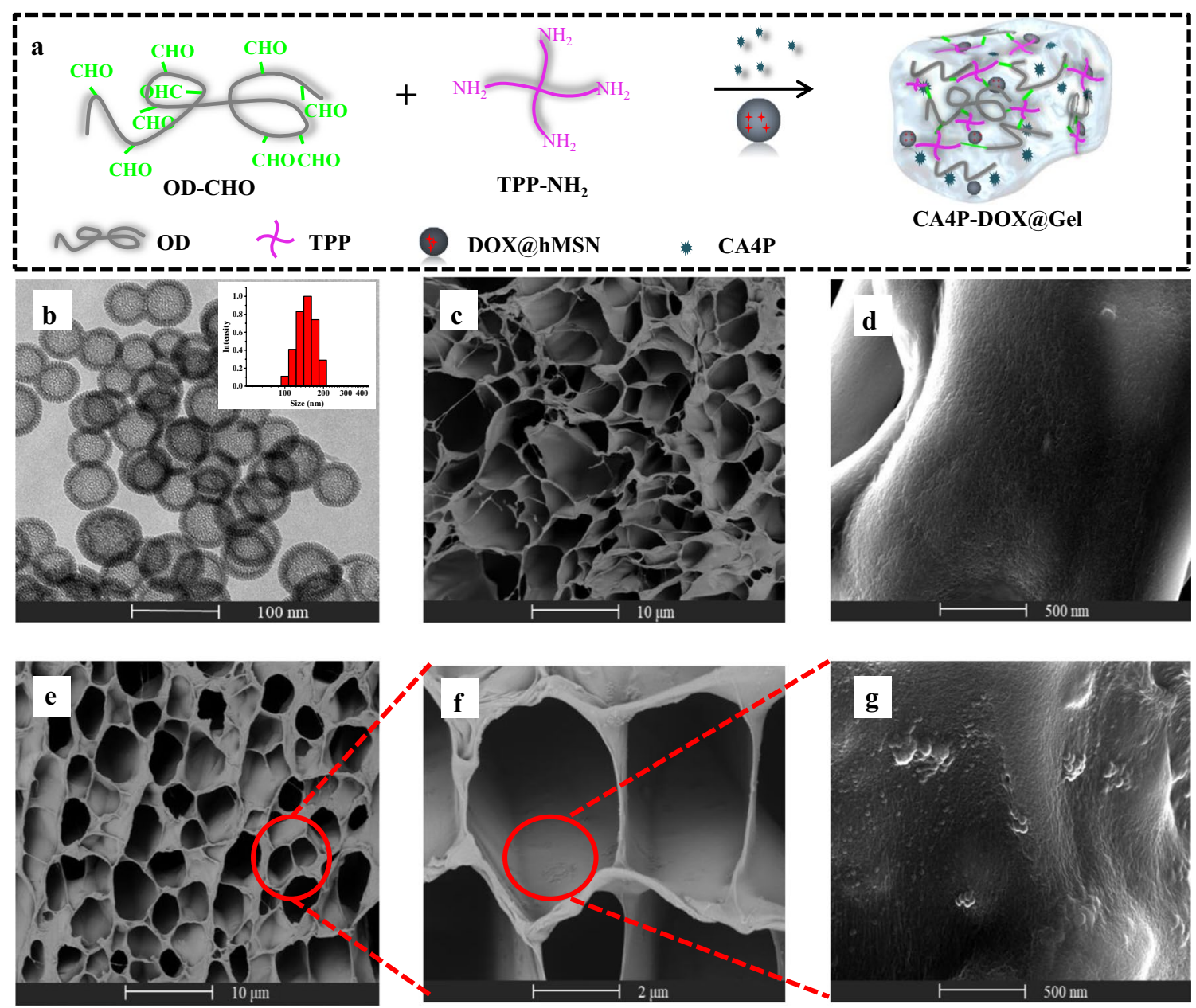

Fig. 2 Schematic diagram of DOX-CA4P@Gel (a), TEM image (insert: DLS measurement) of hMSN (b), SEM images of blank hydrogels (c, d), and SEM images of DOX-CA4P@Gel (e- $\mathbf{g})$

of DOX-CA4P@Gel, indicating the application potential as a tumor-selective drug delivery. The images before and after hydrogel degradation were shown in Fig. 3e, $\mathrm{f}$.

$\mathrm{CA} 4 \mathrm{P}$ and DOX exhibited $\mathrm{pH}$ sensitive and the release profiles were also observed, where faster release rates were shown at acidic condition ( $\mathrm{pH}$ 5.0) compared with that in neutral condition (Fig. 3h, i). CA4P and DOX have different release behavior. As shown in Fig. 3j, CA4P was rapidly released at the early stage and relatively stable after $48 \mathrm{~h}$, while DOX released slowly firstly and then go fast after 48 h. $59.13 \%$ of CA4P was released from Gel at $\mathrm{pH}$ of 6.4 for $48 \mathrm{~h}$, and reached to $63.44 \%$ at $144 \mathrm{~h}$ (only $5.31 \%$ higher than that of $48 \mathrm{~h}$ ). As shown in Fig. 3g, $68.32 \%$ of DOX was release at $\mathrm{pH}$ of 5.0 for $144 \mathrm{~h}$ but only $16.02 \%$ released in a buffer with $\mathrm{pH}$ 7.4. This significant release characteristics of CA4P and DOX form Gel were shown in Fig. 3 j. In the early stage, the release of CA4P was rapid, reaching $71.08 \%$ at $48 \mathrm{~h}$, and slowed down in the later stage, reaching only $78.20 \%$ at $144 \mathrm{~h}$.
As a contrast, DOX released only $24.39 \%$ at $48 \mathrm{~h}$, and increased to $61.60 \%$ at $144 \mathrm{~h}$. The degradation of hydrogel in tumor microenvironment was responsible for the early-stage release of CA4P. Furthermore, the hydrogel capping was also gradually removed from sealing the mesoporous of hMSNs under $\mathrm{pH}$ of 6.4 and 5.0, which accelerated DOX release because of the protonating of the amine group of DOX.

\section{Photodynamic performance of hydrogel}

In order to prove that the photosensitivity of porphyrin was not changed significantly after the incorporation into the hydrogel, DPBF was used as the reagent to quantitatively detect the production of singlet oxygen $\left({ }^{1} \mathrm{O}_{2}\right)$ in solution. The results of the irradiation of the samples in the presence of DPBF (absorption monitored at 410 $\mathrm{nm}$ ) with time are shown in Fig. 4a, where the decrease in DPBF absorption over time for hydrogel compared to TPP, and the decrease was positively correlated with the 

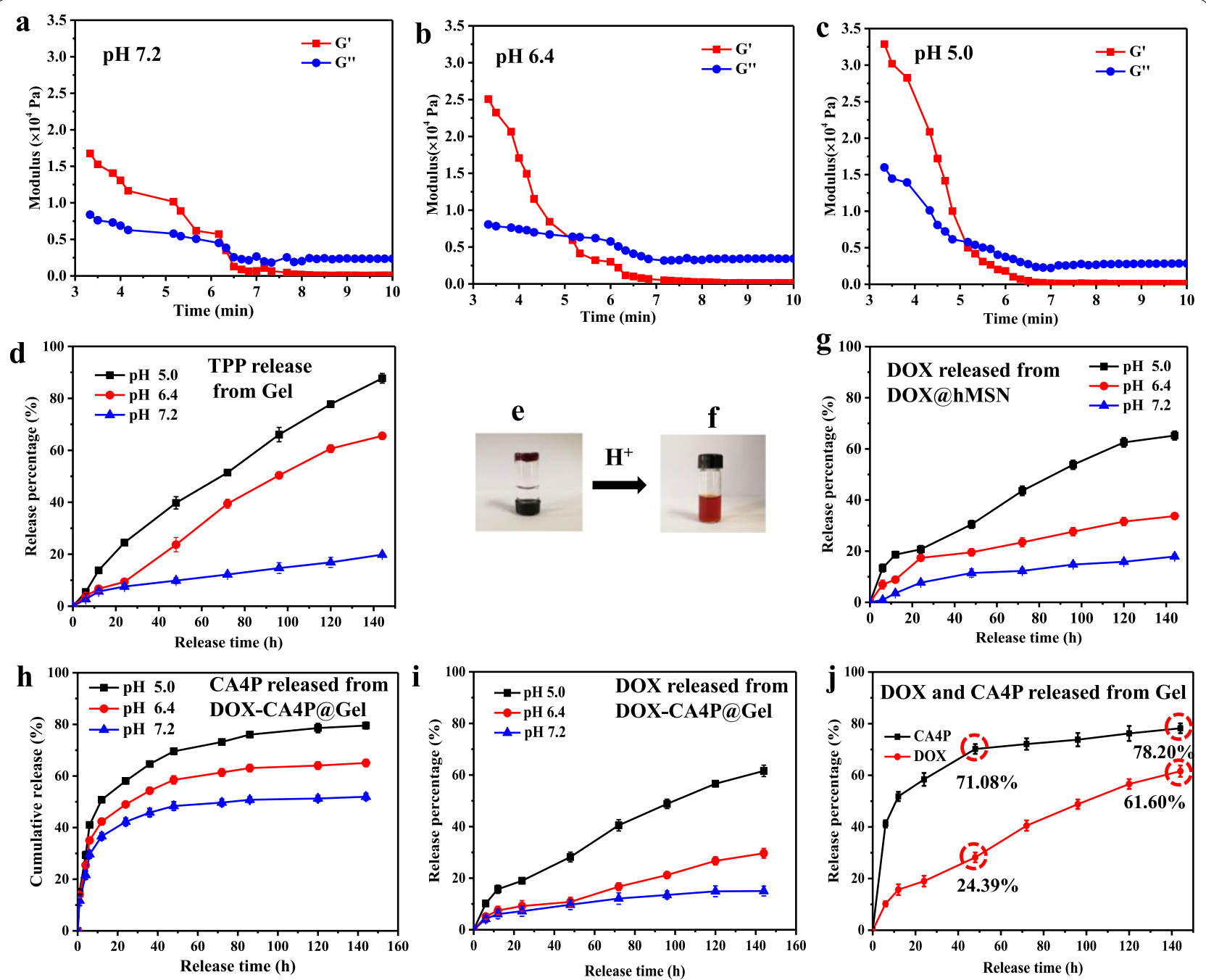

Fig. $3 \mathrm{G}^{\prime}$ and $\mathrm{G}^{\prime \prime}$ of hydrogel in different solution of pH 7.2 (a), pH 6.4 (b), pH 5.0 (c), degradation and release of TPP from hydrogel in PBS with different $\mathrm{pH}$ values $(\mathbf{d})$, macroscopic state of hydrogel under different acidity conditions $(\mathbf{e}, \mathbf{f})$, drug release of DOX from hMSN under different pH medium ( $\mathbf{g}$ ), drug release of CA4P (h) and DOX (i) from hydrogel in different medium respectively, drug release of CA4P, DOX behavior in pH 5.0 (j)

hydrogel concentrations (as shown in Fig. 4b). With TPP $\left(\phi_{\Delta(\mathrm{TPP})}=0.52\right)$ as the control [32], the ${ }^{1} \mathrm{O}_{2}$ yield of hydrogel $\left(808 \mathrm{~nm}, 0.5 \mathrm{~W} / \mathrm{cm}^{2}\right)$ was 0.91 , which may be due to the high viscosity of hydrogel limiting the rotation of porphyrin molecules and avoiding the optical quenching induced by the collision between porphyrin molecules. DCFH-DA was an intracellular singlet oxygen capture agent, and its green fluorescence intensity of cells was directly proportional to the production of ${ }^{1} \mathrm{O}_{2}$. The concentration of After incubation of hydrogel with cells, ${ }^{1} \mathrm{O}_{2}$ detection was conducted. As shown in Fig. 4c, stronger fluorescence emission as compared with those in TPP was present which was consistent with that in PBS.

The extracts of blank hydrogel with different concentrations $(0.05,0.1,0.15$ and $0.2 \mathrm{mg} / \mathrm{mL})$ were incubated with L929 and 4T1 cells for 24, 48 and $72 \mathrm{~h}$, respectively. As shown in Fig. 5a, b, the cellular viabilities of L929 and 4T1 cells were above $85 \%$, indicating that the hydrogel had no obvious toxicity to cells without laser irradiation. However, the cellular viability significantly decreased after laser treatment $\left(\mathrm{NIR}=808 \mathrm{~nm}, 0.5 \mathrm{~W} / \mathrm{cm}^{2}, t=\right.$ $5 \mathrm{~min}$ ). This result shown in Fig. $5 \mathrm{c}$ was particularly significant change at high concentration, that is, at $0.2 \mathrm{mg} /$ $\mathrm{ml}$, the cell viability of the hydrogel group without laser assistance was $92.43 \%$, while that of with laser group was reduced to $65.31 \%$, with a significant difference $(p<0.05)$, suggesting that hydrogel has an obvious photodynamic killing effect on 4T1 cells. In order to further intuitively demonstrate the PDT effect of hydrogel on cancer cells, the live/dead cells staining with calcein AM and PI was 

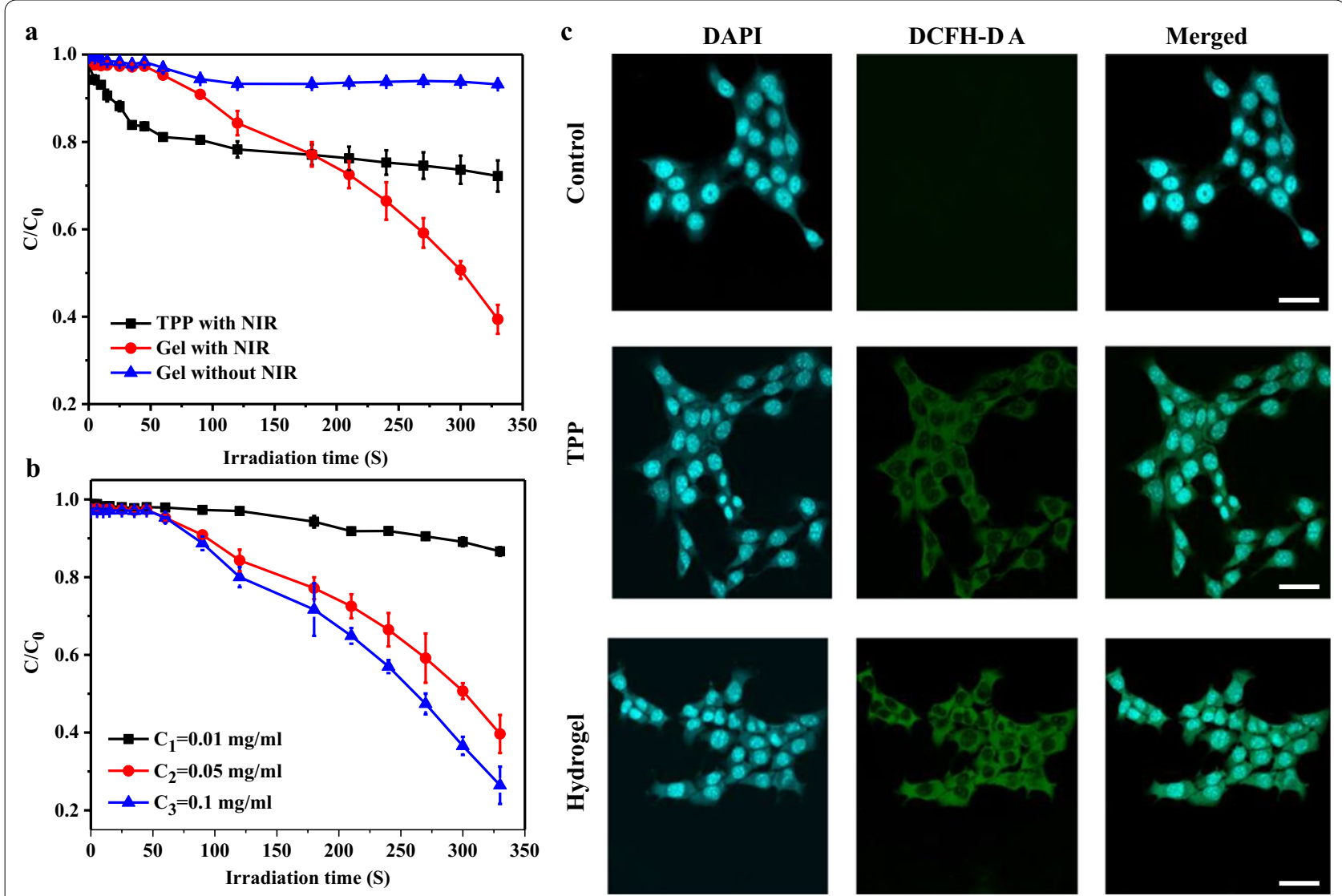

Fig. $4{ }^{1} \mathrm{O}_{2}$ generation measurement of hydrogel $(\mathbf{a}),{ }^{1} \mathrm{O}_{2}$ generation depended on concentrations of hydrogel $(\mathbf{b}),{ }^{1} \mathrm{O}_{2}$ generation in cancer cells detected by confocal microscopy (c). scale bar: $50 \mu \mathrm{m}$

carried out. The fluorescence imaging results in Fig. 5d indicated that the proportion of dead cells increased significantly with the elevated concentrations of the hydrogel extract. Moreover, with the increase of irradiation time (showed in Additional file 1: Figure S4), the proportion of dead cells also increased significantly, indicating that the photodynamic treatment effect of hydrogel was obvious concentration and time dependent.

\section{In vitro chemo-photodynamic therapy effect of DOX-CA4P@Gel}

Gel, DOX@Gel, CA4P@Gel, and DOX-CA4P@Gel $(0.1 \mathrm{mg} / \mathrm{ml})$ were respectively incubated with $4 \mathrm{~T} 1$ cells for $48 \mathrm{~h}$, and the cell viabilities were measured with or without laser irradiation $\left(808 \mathrm{~nm}, 0.5 \mathrm{~W} / \mathrm{cm}^{2}, 5 \mathrm{~min}\right)$. PBS was used as the control. As showed in Additional file 1: Figure S5, the cell viability of both Gel and DOX@ Gel groups were above $90 \%$, indicating that no obvious DOX was released during this period. In contrast, the cell viability of both CA4P@Gel and DOX-CA4P@Gel groups were lower than $70 \%$. Furthermore, cell viability in CA4P@Gel significantly decreased with time lasting to
$48 \mathrm{~h}$, indicating that CA4P could be continuously released within $48 \mathrm{~h}$. With laser irradiation, all the groups including Gel+NIR, DOX@Gel+NIR, CA4P@Gel+NIR and DOX-CA4P@Gel+NIR, exhibited enhanced cell killing effect. Especially, the cell activity of DOX-CA4P@ Gel+NIR group was lower than $40 \%$, which verified that DOX-CA4P@Gel has significant combinational effect of photodynamic/sequential chemotherapy.

This result was also verified in a flow cytometry assay. As shown in Fig. 6, without laser irradiation, the cell viability (95.5\%) of DOX@Gel was not significantly different from that of Gel (95.8\%), while CA4P@Gel and DOX-CA4P@Gel decreased to 79.5\% and 66.6\%, respectively. This is due to DOX-CA4P@Gel mainly showed the release of CA4P within $24 \mathrm{~h}$, while DOX did not show obvious tumor killing effect because of its late release. Furthermore, after the same sample treatment, the cell viability with laser irradiation was significantly lower than that of the non-irradiated group, especially the cell viability of DOX-CA4P@Gel+NIR group decreased from 66.6 to $53.3 \%$, reflecting obvious combined treatment effect. 


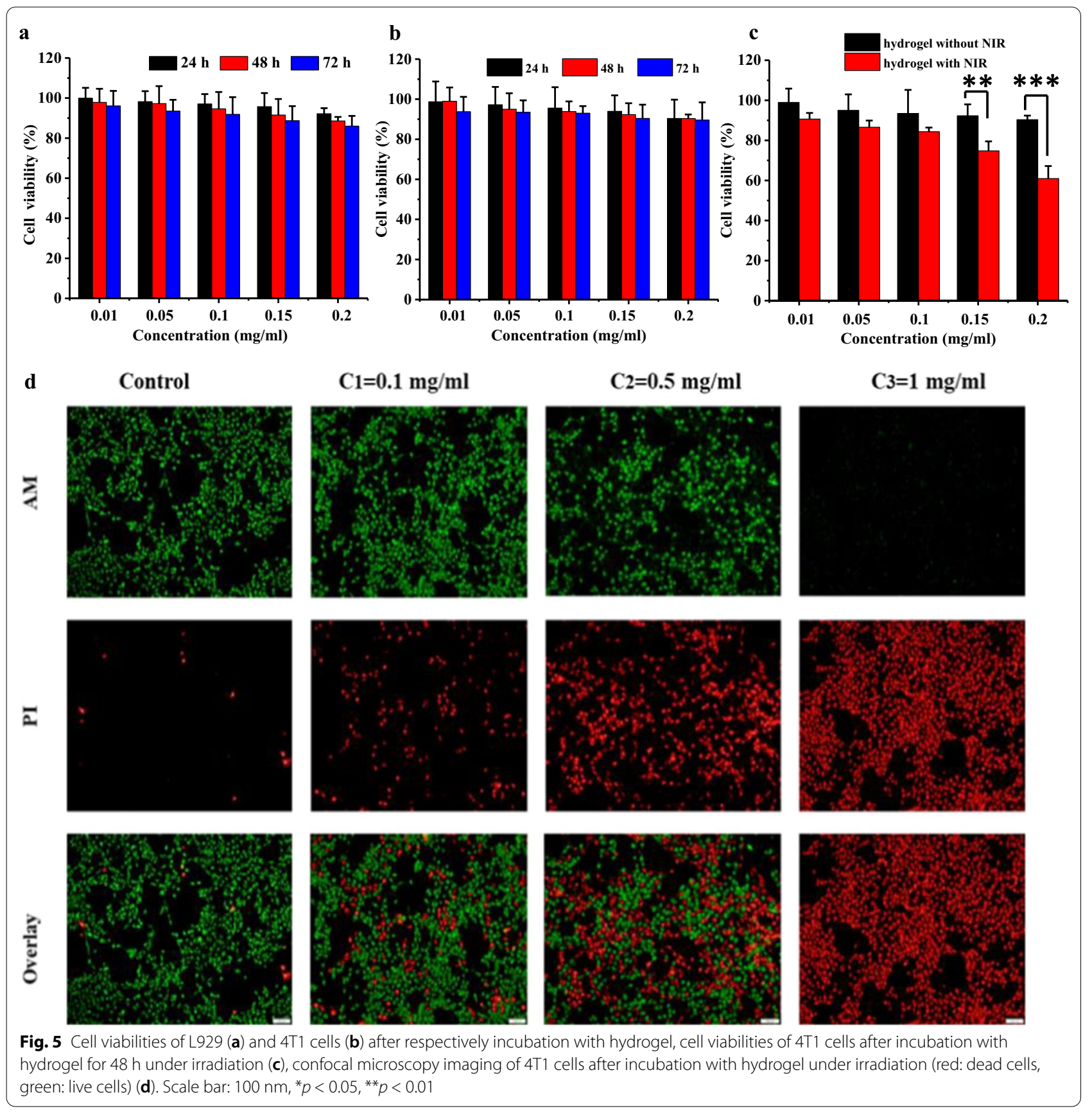

Comparing the cell viability of groups (longitudinal groups) without laser assistance, results showed that after 24 h of co-incubation with 4T1 cells, only CA4P@ Gel (79.5\%) and DOX-CA4P@Gel (66.6\%) was significantly lower than that of control group (95.3\%), while DOX@Gel group was not significantly different from the control group, which proved that early release of CAP4P from DOX-CA4P@Gel was the major reason for cell toxicity before $24 \mathrm{~h}$. Comparing the Gel groups (lateral groups), it was found that the cell viability with laser assistance was significantly lower than that of the without laser assistance group, especially DOX-CA4P@Gel+NIR was only $50 \%$.

\section{Combinational therapy of DOX-CA4P@Gel}

The 4T1 tumor-bearing mice were respectively treated by a single intratumor injection administration of PBS, Free DOX/CA4P (mixture of $0.4 \mathrm{mg}$ DOX and $1 \mathrm{mg}$ 


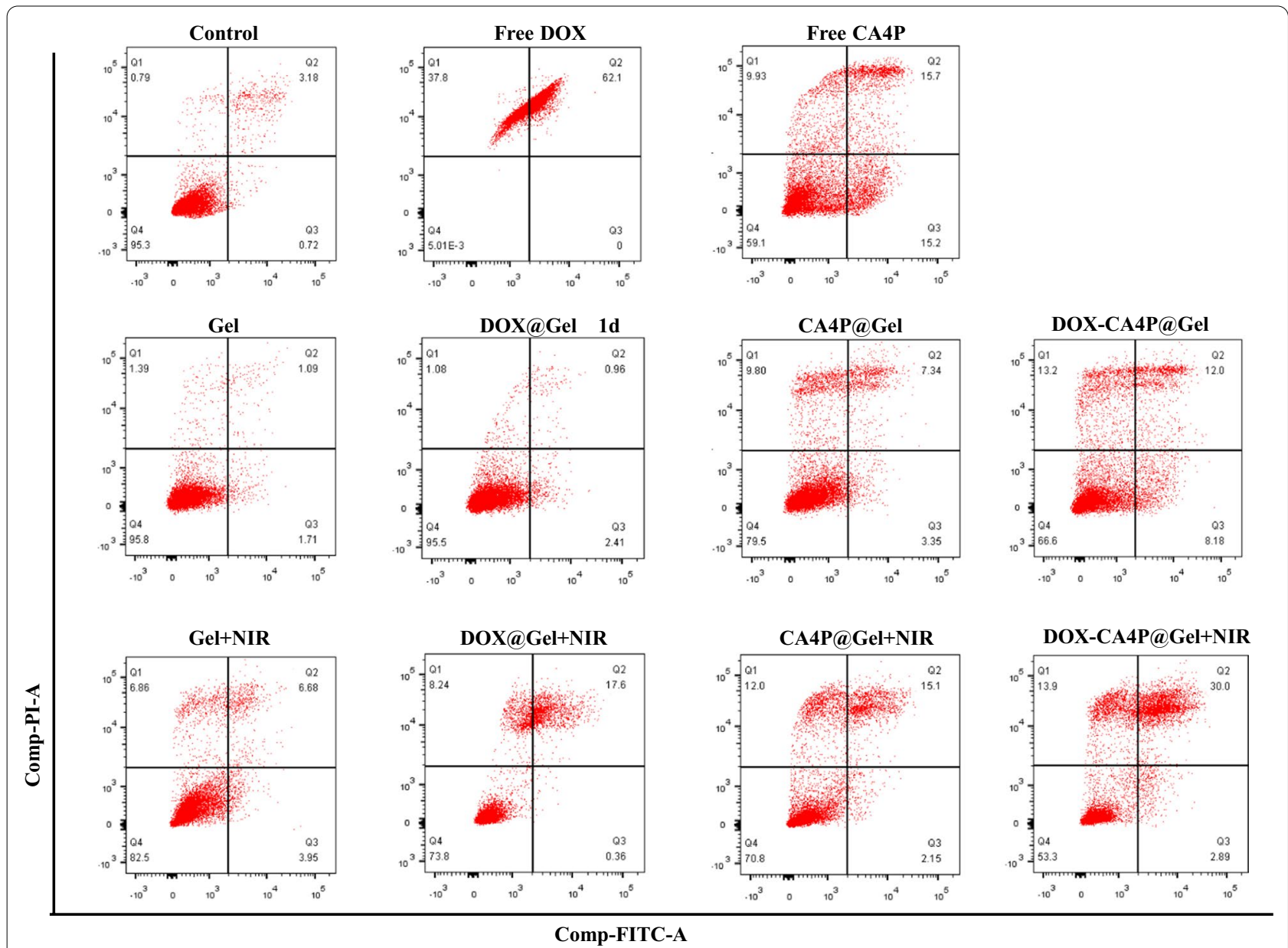

Fig. 6 Flow cytometry analysis of $4 \mathrm{~T} 1 \mathrm{cell}$ s after $24 \mathrm{~h}$ of incubation with different groups. PBS was used as the negative control group, and free DOX/CA4P was used as the positive control group ( $\left.808 \mathrm{~nm}, 0.5 \mathrm{~W} / \mathrm{cm}^{2}, 5 \mathrm{~min}\right)$

CA4P), Gel, CA4P@Gel, DOX@Gel, DOX-CA4P@Gel, Gel+NIR, CA4P@Gel+NIR, DOX@Gel+NIR, DOXCA4P@Gel+NIR, where NIR was illuminated for $5 \mathrm{~min}$ every other day. The body weight, the volume and weight of the tumor were recorded every day, and the tumor inhibition rate was calculated with PBS as the control group. As can be seen from Fig. $7 \mathrm{a}-\mathrm{c}$, the tumor in the CA4P@Gel group grew slowly in the first 6 days and then accelerated, while the tumor in the DOX@Gel group grew rapidly in the first 6 days but slowed down significantly within 6-12 days, which may be explained by the sequential release of CA4P and DOX. With the assistance of laser, the comparison of treatment results between the DOX-CA4P@Gel +NIR group and other groups showed that the tumors in the DOX-CA4P@Gel +NIR group were the smallest and significantly different from those in other groups $(p<0.01)$, indicating that sequential delivery therapy combined with photodynamic therapy had a significant impact on tumor growth. It should be noted that after 18 days of treatment, tumor growth was accelerated in the DOX/CA4P group, with tumor volume greater than that of CA4P-DOX@Gel, which may be due to the fact that CA4P stimulated the formation of tumor vascular collateral after a single dose, thus accelerating tumor growth. In the DOX-CA4P@Gel system, CA4P plays its role first, followed by DOX and PDT. Sequential drug release combined with photodynamic therapy can effectively inhibit tumor growth to a certain extent. The therapeutic effect of each group was also evaluated by measuring tumor weight, and the results were shown in Fig. $7 \mathrm{~d}$. The results showed that the tumor weight of the DOX-CA4P@Gel +NIR group was the lowest, which was consistent with that of tumor volume growth curves. As showed in Fig. 7e, the calculated tumor inhibition rate was about 70\% for DOX-CA4P@Gel +NIR group, which was significantly different from other groups $(p<0.01)$.

The expression of caspase 3 and DOX fluorescence intensity were used to characterize the location of 

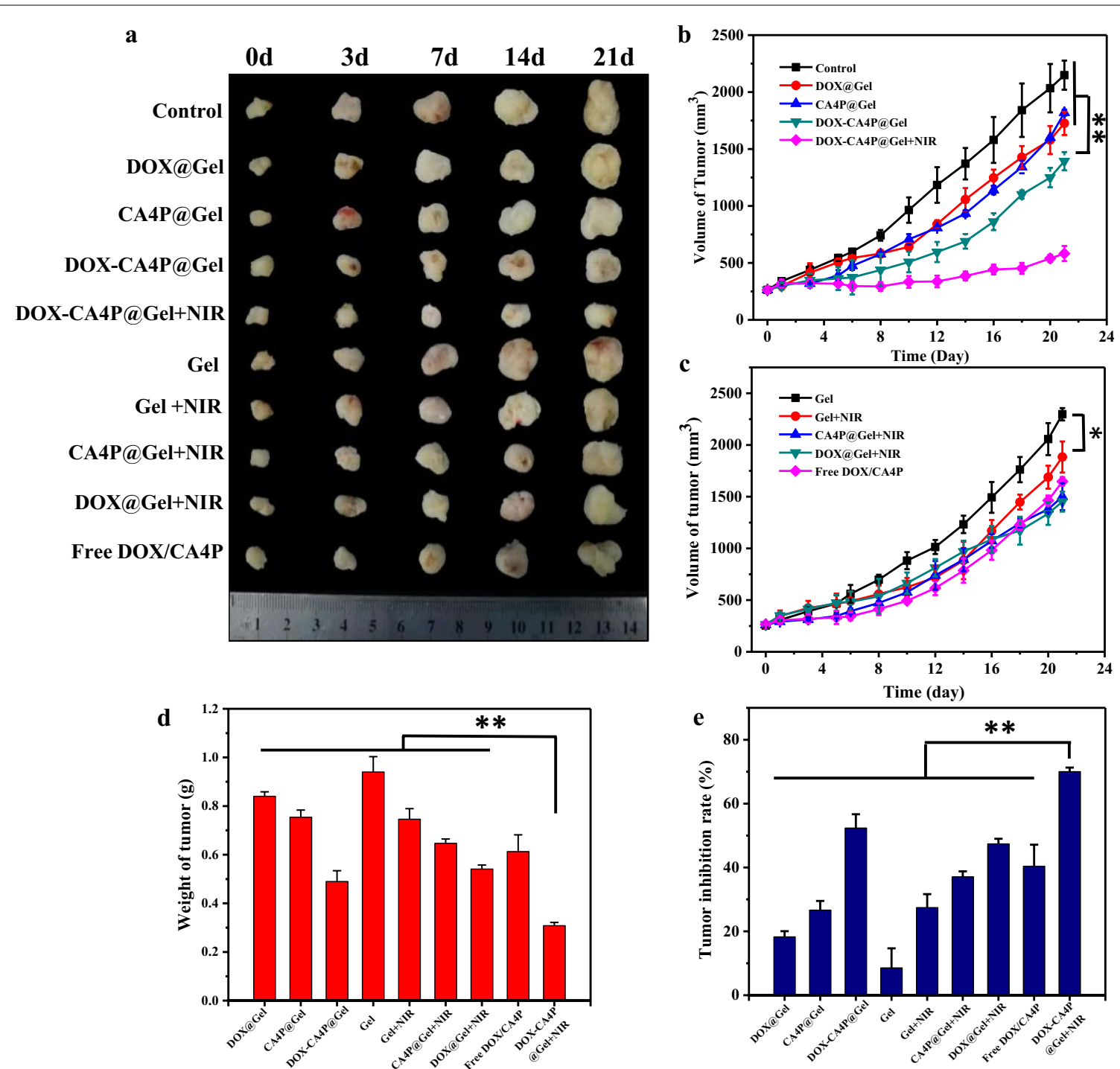

Fig. 7 Isolated tumor during the treatment cycle (a), tumor volume of the mice during the treatment cycle (b, c), tumor weight (d) and tumor growth inhibition rate (e) post-treated by different samples for three weeks. ${ }^{*} p<0.05,{ }^{* *} p<0.01$

CA4P and DOX. CA4P can specifically bind to vascular growth factor and prevent its interaction with the receptor, thereby causing apoptosis. The activity of caspase 3 reflects the apoptotic status of tumor cells. At different time points, the caspase 3 activity (apoptotic inducing factor) in the tumors of the DOX-CA4P@Gel group was detected by immunohistochemistry. Results in Fig. 8 showed that compared with that in control group, the caspase 3 expression was significantly up-regulated, reaching the peak on the 3rd day, and gradually went down in the subsequent treatment period. DOX release and accumulation in tumor sections of the DOX-CA4P@ Gel group at different time points $(2,6,10,14$ days) were measured by the fluorescence properties of DOX.
The results showed that DOX fluorescence in tumor tissues was extremely weak at the initial stage of treatment (within 2 days), suggesting DOX-CA4P@Gel accumulating in the tumor. With the extension of time (at the 6th days), it retained in tumor for more than $14 \mathrm{~d}$.

\section{In vivo safety evaluation}

To evaluate the possible systemic toxicity, body weigh variation and $H \& E$ staining was carried out. Additional file 1: Figure S6 showed that there was no significant statistical difference in the body weight of mice between the control group and each treatment group, indicating that the therapeutic agent had no significant toxicity in vivo. $H \& E$ staining of the representative tissue sections, 


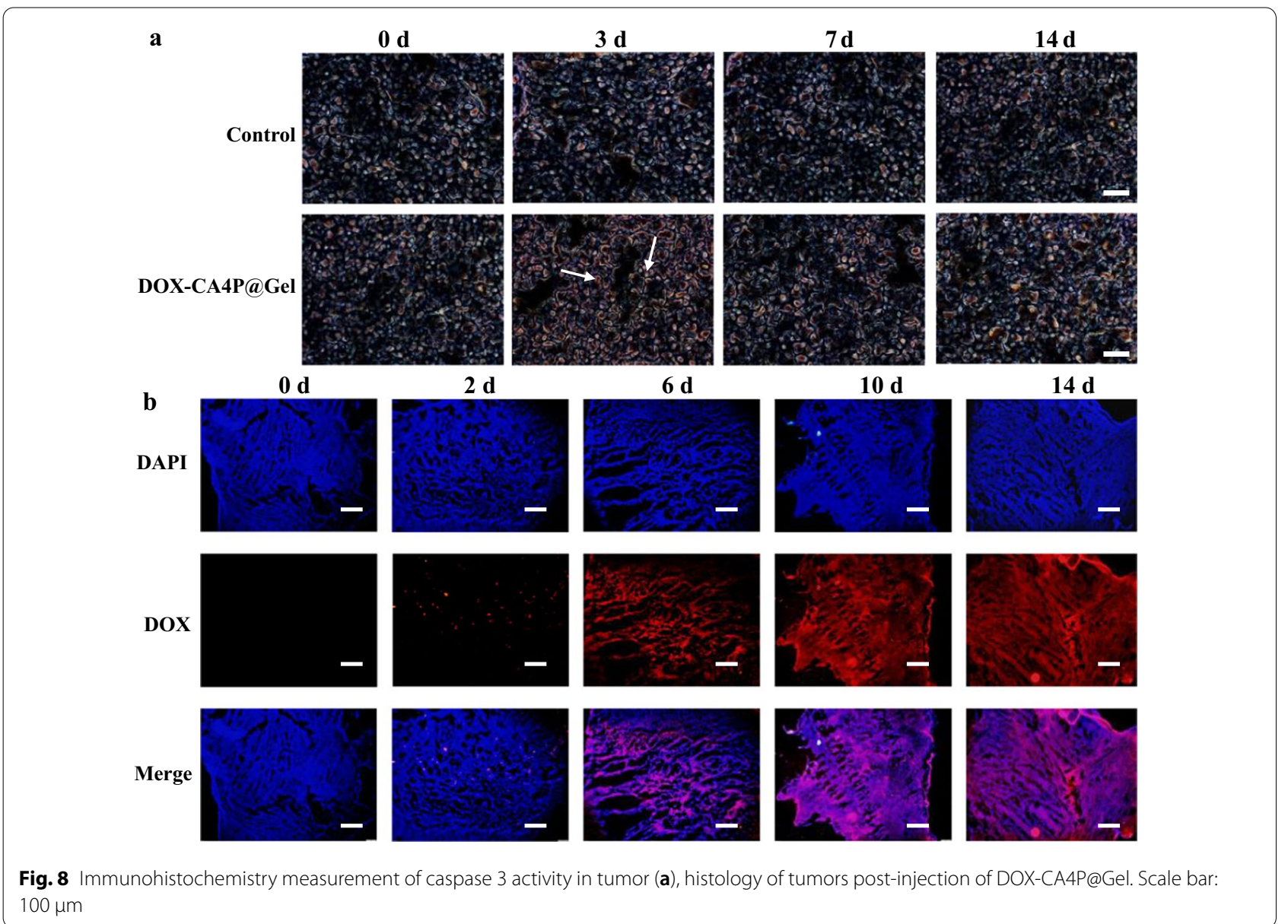

including heart, liver, spleen, lung, and kidney was carried out for the mice treated with PBS and different Gel conjugates at day 21. As shown in Additional file 1: Figure S7, no observable systemic toxicity was noted in all the organs of the tumor-bearing mice treated with the drugloaded hydrogel groups.

\section{Conclusions}

With biocompatible dextran oxide, chitosan, porphyrin and hMSN as the starting materials, a dual drug carrying system DOX-CA4P@Gel was constructed, which could be injected in tumor and play therapeutic roles in situ. Good biocompatibility DOX-CA4P@Gel was verified by cell tests and H\&E staining. Studies on the properties of the materials show that Gel can be degraded slowly under tumor microenvironment, and DOX and CA4P released sequentially for tumor therapy at different time. Animal model experiments showed that DOX-CA4P@Gel+NIR could effectively enhance therapy effect due to the sequential drugs release and photodynamic performance. The DOX-CA4P@Gel constructed in this work is simultaneously injectable, biodegradable, sequentially releasable and photodynamic, providing a feasible route to reduce drug resistance and improve cancer therapy efficiency.

\section{Supplementary Information}

The online version contains supplementary material available at https://doi. org/10.1186/s12951-021-01066-1.

Additional file 1: Figure S1. Zeta potential of hMSN, DOX@hMSN-NH2, CA4P@Gel and DOX-CA4P@Gel. Figure S2.XPS survey spectra of hMSN, Gel and CA4P-DOX@Gel. Figure S3. Visible spectra of DOX, TPP and CA4P. Figure S4. Fluorescence images of 4T1 cells after incubation with hydrogel with irradiation assistance (red-dead cells, green-live cells). Scale bar: $100 \mu \mathrm{m}$. Figure $\mathbf{S 5}$. Cell viability of $4 \mathrm{~T} 1$ cells post different treatment. ${ }^{* * *} p<0.001$. Figure $\mathbf{S 6}$. Body weight variation of mice post different treatment. Figure S7. Histolodical analysis of the major organs of mice treated with various drug formulations at day 21 (scale bar is $100 \mu \mathrm{m}$ ). Table S1. The degree of aldehyde affected by the oxidation time by sodium periodate. Table S2. Effect of chitosan on hydrogel formation and degradation.

\section{Acknowledgements}

Some structural performances were characterized by Dr. Ma from Beijing University of Chemical Technology, and we would like to thank her for his help. 


\section{Authors' contributions}

$Y Z$ performed the data analyses and wrote the manuscript. $Y Z, L Z$ and $Z Z$ performed the experiment. SS and YM contributed significantly to analysis and manuscript preparation. Hao helped perform the analysis with constructive discussions. DY contributed to the conception of the study. All authors read and approved the final manuscript.

\section{Funding}

National Natural Science Foundation of China (61901405), Natural Science Foundation of Jiangsu Province, China (BK20201156), Xuzhou Natural Science Foundation, China (KC18108 and KC19066) and Excellent Talents Scientific Research Project (D2019026), and Founded by China Postdoctoral Science Foundation (2020M671608).

\section{Availability of data and materials}

All data analyzed during this study are included in this published article.

\section{Declarations}

\section{Ethics approval and consent to participate}

Not applicable.

\section{Consent for publication}

All authors of this work consent to the publication of the paper.

\section{Competing interests}

The authors declare that they have no known competing financial interests or personal relationships that could have appeared to influence the work reported in this paper.

\section{Author details \\ 'Jiangsu Key Laboratory of New Drug Research and Clinical Pharmacy, Xuzhou 221000, Jiangsu, China. \\ Received: 6 August 2021 Accepted: 28 September 2021 \\ Published online: 23 October 2021} Xuzhou Medical University, Xuzhou 221004, Jiangsu, China. ${ }^{2}$ Medical School, Nanjing University, Nanjing 210093, Jiangsu, China. ${ }^{3}$ Xuzhou Air Force College,

\section{References}

1. Wu X, Wu Y, Ye H, Yu S, He C, Chen X. Interleukin-15 and cisplatin coencapsulated thermosensitive polypeptide hydrogels for combined immuno-chemotherapy. J Control Release. 2017;255:81-93.

2. Dong X, Chen H, Qin J, Wei C, Liang J, Liu T, Kong D, Lv F. Thermosensitive porphyrin-incorporated hydrogel with four-arm PEG-PCL copolymer (II): doxorubicin loaded hydrogel as a dual fluorescent drug delivery system for simultaneous imaging tracking in vivo. Drug Deliv. 2017;24:641-50.

3. Belali S, Savoie H, O'Brien JM, Cafolla AA, O'Connell B, Karimi AR, Boyle RW, Senge MO. Synthesis and characterization of temperature-sensitive and chemically cross-linked poly(N-isopropylacrylamide)/photosensitizer hydrogels for applications in photodynamic therapy. Biomacromol. 2018;19:1592-601.

4. Burt LM, Ying J, Poppe MM, Suneja G, Gaffney DK. Risk of secondary malignancies after radiation therapy for breast cancer: comprehensive results. Breast. 2017;35:122-9.

5. Chen H, Zhao Y. Applications of light-responsive systems for cancer theranostics. ACS Appl Mater Interfaces. 2018;10:21021-34.

6. Zhang Q, Li L. Photodynamic combinational therapy in cancer treatment. J Buon. 2018;23:561-7.

7. Lieshout HF, Bots CP. The effect of radiotherapy on dental hard tissue-a systematic review. Clin Oral Investig. 2014;18:17-24.

8. Jiang J, Shen N, Song WT, Yu HY, Sakurai K, Tang ZH, Li G. Combretastatin A4 nanodrug combined plerixafor for inhibiting tumor growth and metastasis simultaneously. Biomater Sci. 2019;7:5283-91.

9. Brown AW, Holmes T, Fisher M, Tozer GM, Harrity JPA, Kanthou C. Evaluation of sydnone-based analogues of combretastatin A-4 phosphate (CA4P) as vascular disrupting agents for use in cancer therapy. ChemMedChem. 2018;13:2618-26.
10. Zhu JF, Hu MY, Qiu LY. Drug resistance reversal by combretastatin-A4 phosphate loaded with doxorubicin in polymersomes independent of angiogenesis effect. J Pharm Pharmacol. 2017;69:844-55.

11. Zhu JF, Xu XP, Hu MY, Qiu LY. Co-encapsulation of combretastatin-A4 phosphate and doxorubicin in polymersomes for synergistic therapy of nasopharyngeal epidermal carcinoma. J Biomed Nanotechnol. 2015;11:997-1006.

12. Bardajee GR, Hosseini SS, Ghavami S. Embedded of nanogel into multiresponsive hydrogel nanocomposite for anticancer drug delivery. J Inorg Organomet P. 2018;28:2196-205.

13. Fu JJ, Chen MY, Li JX, Zhou JH, Xie SN, Yuan P, Tang B, Liu CC. Injectable hydrogel encapsulating $\mathrm{Cu}_{2} \mathrm{MnS}_{2}$ nanoplates for photothermal therapy against breast cancer. J Nanobiotechnol. 2018;16(1):83.

14. Zhao LL, Zhu LJ, Liu FY, Liu CY, Shan-Dan, Wang Q, Zhang CL, Li JL, Liu JG, Qu XZ, Yang ZZ. pH triggered injectable amphiphilic hydrogel containing doxorubicin and paclitaxel. Int J Pharmaceut. 2011;410:83-91.

15. Gao G, Jiang YW, Jia HR, Wu FG. Near-infrared light-controllable ondemand antibiotics release using thermo-sensitive hydrogel-based drug reservoir for combating bacterial infection. Biomaterials. 2019;188:83-95.

16. Park J, Pramanick S, Park D, Yeo J, Lee J, Lee H, Kim WJ. Therapeutic-gasresponsive hydrogel. Adv Mater. 2017;29:1702859.

17. Kim DY, Kwon DY, Kwon JS, Park JH, Park SH, Oh HJ, Kim JH, Min BH, Park K, Kim MS. Synergistic anti-tumor activity through combinational intratumoral injection of an in-situ injectable drug depot. Biomaterials. 2016;85:232-45.

18. Bera K, Maiti S, Maity M, Mandal C, Maiti NC. Porphyrin-gold nanomaterial for efficient drug delivery to cancerous cells. ACS Omega. 2018;3:4602-19.

19. Belali S, Karimi AR, Hadizadeh M. Cell-specific and pH-sensitive nanostructure hydrogel based on chitosan as a photosensitizer carrier for selective photodynamic therapy. Int J Biol Macromol. 2018;110:437-48.

20. Gonzalez-Delgado JA, Castro PM, Machado A, Araujo F, Rodrigues F, Korsak B, Ferreira M, Tome JPC, Sarmento B. Hydrogels containing porphyrin-loaded nanoparticles for topical photodynamic applications. Int J Pharmaceut. 2016;510:221-31.

21. Dong JJ, Yao XY, Sun SA, Zhong YY, Qian CT, Yang DZ. In vivo targeting of breast cancer with a vasculature-specific GQDs/hMSN nanoplatform. RSC Adv. 2019;9:11576-84.

22. Zhao X, Li P, Guo BL, Ma PX. Antibacterial and conductive injectable hydrogels based on quaternized chitosan-graft-polyaniline/oxidized dextran for tissue engineering. Acta Biomater. 2015;26:236-48.

23. Yan SF, Wang TT, Feng L, Zhu J, Zhang KX, Chen XS, Cui L, Yin JB. Injectable in situ self-cross-linking hydrogels based on poly(L-glutamic acid) and alginate for cartilage tissue engineering. Biomacromol. 2014;15(12):4495-508.

24. Yang DZ, Yao XY, Dong JJ, Wang N, Du Y, Sun SA, Gao LP, Zhong YY, Qian $\mathrm{CT}$, Hong $\mathrm{H}$. Design and investigation of core/shell GQDs/hMSN nanoparticles as an enhanced drug delivery platform in triple-negative breast cancer. Bioconjugate Chem. 2018;29:2776-85.

25. Venegas B, Zhu WW, Haloupek NB, Lee J, Zellhart E, Sugar IP, Kiani MF, Chong PLG. Cholesterol superlattice modulates CA4P release from liposomes and CA4P cytotoxicity on mammary cancer cells. Biophys J. 2012;102:2086-94.

26. Jin S, Wan JX, Meng LZ, Huang XX, Guo J, Liu L, Wang CC. Biodegradation and toxicity of protease/redox/pH stimuli-responsive peglated pmaa nanohydrogels for targeting drug delivery. ACS Appl Mater Interfaces. 2015;7:19843-52.

27. De Baroid AT, McCoy CP, Craig RA, Carson L, Andrews GP, Jones DS, Gorman SP. Optimization of singlet oxygen production from photosensitizer-incorporated, medically relevant hydrogels. J Biomed Mater Res B. 2017;105:320-6.

28. National Research Council. Guide for the care and use of laboratory animals. 8th ed. Washington DC: The National Academies Press; 2011.

29. Dai TJ, Wang CP, Wang YQ, Xu W, Hu JJ, Cheng YY. A nanocomposite hydrogel with potent and broad-spectrum antibacterial activity. ACS Appl Mater Interfaces. 2018;10:15163-73.

30. Xia LY, Zhang XD, Cao M, Chen Z, Wu FG. Enhanced fluorescence emission and singlet oxygen generation of photosensitizers embedded in injectable hydrogels for imaging-guided photodynamic cancer therapy. Biomacromolecules. 2017;18:3073-81. 
31. Wang JP, Liu L, You Q, Song YL, Sun Q, Wang YD, Cheng Y, Tan FP, Li N. Allin-one theranostic nanoplatform based on hollow mosx for photothermally-maneuvered oxygen self-enriched photodynamic therapy. Theranostics. 2018;8:955-71.

32. Korínek M, Dědic R, Molnár A, Svoboda A, Hála J. A comparison of photosensitizing properties of meso-tetraphenylporphin in acetone and in dimethyl sulfoxide. J Mol Struct. 2005;744:727-31.

\section{Publisher's Note}

Springer Nature remains neutral with regard to jurisdictional claims in published maps and institutional affiliations.
Ready to submit your research? Choose BMC and benefit from:

- fast, convenient online submission

- thorough peer review by experienced researchers in your field

- rapid publication on acceptance

- support for research data, including large and complex data types

- gold Open Access which fosters wider collaboration and increased citations

- maximum visibility for your research: over $100 \mathrm{M}$ website views per year

At BMC, research is always in progress.

Learn more biomedcentral.com/submissions 